\title{
Medication Adherence, Depressive Symptoms, and Cardiac Event-Free Survival in Patients with Heart Failure
}

\author{
Jia-Rong Wu, PhD, RN [Assistant Professor], \\ University of North Carolina at Chapel Hill, School of Nursing
}

Terry A. Lennie, PhD, RN, FAAN [Professor and Director of the PhD Program], University of Kentucky, College of Nursing

Rebecca L. Dekker, PhD, APRN [Assistant Professor],

University of Kentucky, College of Nursing

Martha J. Biddle, PhD, APRN, CCNS [Assistant Professor], and

University of Kentucky, College of Nursing

Debra K. Moser, DNSc, RN, FAAN [Professor and Gill Endowed Chair of Nursing]

University of Kentucky, College of Nursing

\section{Abstract}

Background-Medication nonadherence and depressive symptoms predict hospitalization and death in patients with heart failure (HF). Depressed patients have lower medication adherence than non-depressed patients. However, the predictive power of the combination of medication adherence and depressive symptoms for hospitalization and death has not been investigated in patients with HF.

Objective-To explore the combined influence of medication adherence and depressive symptoms for prediction of cardiac event-free survival in patients with HF.

Method-We monitored medication adherence in $216 \mathrm{HF}$ patients who completed the Patient Health Questionnaire-9 (PHQ-9) at baseline. Medication adherence was measured objectively using Medication Event Monitoring System (MEMS). Patients were followed for up to $31 / 2$ years to collect data on cardiac event(s). Survival analyses were used to compare cardiac event-free survival between/among groups.

Results-The risk of experiencing a cardiac event for patients with medication nonadherence and depressive symptoms was 5 times higher compared to those who were medication adherent without depressive symptoms. The risk of experiencing a cardiac event for patients with only one risk factor was 1.2-1.3 times that of those with neither risk factor.

Conclusion-Medication nonadherence and depressive symptoms had a negative synergistic effect on cardiac event-free survival in patients with HF.

() 2013 Elsevier Inc. All rights reserved.

Address for correspondence: Jia-Rong Wu, PhD, RN, University of North Carolina at Chapel Hill, School of Nursing, 435 Carrington Hall, CB \# 7460, Chapel Hill, NC 27599-7460, Phone 919-966-8057, Fax 919-843-9900, jiarongw @ email.unc.edu.

Publisher's Disclaimer: This is a PDF file of an unedited manuscript that has been accepted for publication. As a service to our customers we are providing this early version of the manuscript. The manuscript will undergo copyediting, typesetting, and review of the resulting proof before it is published in its final citable form. Please note that during the production process errors may be discovered which could affect the content, and all legal disclaimers that apply to the journal pertain.

Disclosures

The authors have nothing to disclose. 


\section{Keywords}

medication adherence; depressive symptoms; heart failure; outcomes

\section{Introduction}

Heart failure (HF) is a chronic syndrome with high incidence and prevalence, and high rates of morbidity and mortality. ${ }^{1}$ In a meta-analysis, Rutledge and associates reported that more than one fifth $(21.5 \%)$ of patients with HF suffer from clinically significant depression ${ }^{2}$ that adversely affects health-related outcomes (e.g., more hospitalizations and death). ${ }^{2,3}$ Emergency department (ED) visits, hospitalizations and death are higher in HF patients with depressive symptoms compared to those without depressive symptoms. ${ }^{2,3,4}$ Specifically, patients with HF and depressive symptoms have twice the risk of ED visits and a $29 \%$ increase in health care costs compared to those without depressive symptoms. ${ }^{2}$ Faris and colleagues $^{3}$ examined a cohort of 396 patients with HF and also found that clinically depressed patients were three times more likely to die compared to non-depressed patients.

Medication nonadherence ${ }^{4-10,16}$ also has been shown to independently predict worse event-free survival and higher rates of ED visits, hospitalizations, and death in patients with HF. However, medication adherence rates in this population are low, between $40-60 \%{ }^{11,12}$ Patients with depressive symptoms are more likely to be nonadherent to their prescribed medication than those without depressive symptoms. ${ }^{13}$ The power of the combination of medication adherence and depressive symptoms for prediction of cardiac event-free survival has not been previously investigated in patients with HF. Accordingly, the purpose of this study was to explore the predictive power of the combination of medication adherence and depressive symptoms for cardiac event-free survival in patients with HF.

\section{Methods}

\section{Study Design}

This was a secondary data analysis of two prospective studies in which patients with HF were enrolled to measure medication adherence (measured objectively using the Medication Event Monitoring System [MEMS]) and cardiac event-free survival. ${ }^{10,14}$ Both studies used identical inclusion and exclusion criteria. The first study tested the mediating effects of nonadherence on the association between depression and HF patient outcomes. The second study was a randomized controlled trial to determine the effect of an intervention on morbidity and mortality outcomes. In this data analysis, we examined the relationships among medication adherence, depressive symptoms, and cardiac event-free survival in patients with HF.

\section{Samples and Setting}

Detailed eligibility criteria and recruitment methods have been published previously. 11, 14, 15, 21 Briefly, patients were recruited from both outpatient cardiology clinics and inpatient cardiology units. Patients with and without systolic dysfunction were included if they had a confirmed diagnosis of chronic HF and were prescribed the same dose of HF medications for at least 3 months. Patients who had obvious cognitive impairment or a co-existing terminal illness were excluded.

\section{Measurement of Variables}

Medication adherence-Medication adherence was assessed daily for 1-3 months (3 months for the first study and 1 month for the second study) using the MEMS (AARDEX ${ }^{\circledR}$ - 
USA, Union City, CA). The MEMS registered the date and time of cap openings. From the MEMS data, medication adherence was calculated from number of days the correct number of doses were taken during the monitoring period /total days during the monitoring period* $100 \% .{ }^{16}$ Patients who took the correct number of doses on at least $88 \%$ of days were defined as medication adherent, while all others were as nonadherent. This cutpoint was chosen based on a study demonstrating that adherence at or above $88 \%$ predicted better event-free survival. ${ }^{11}$

Depressive symptoms-Depressive symptoms were measured using the Patient Health Questionnaire-9 (PHQ-9). ${ }^{17,} 18$ The PHQ-9 consists of nine items on a scale rated from 0 (not at all) to 3 (nearly every day). The instrument is scored by adding the item ratings. Total scores of the PHQ-9 can range from 0 to 27; higher scores indicate worse depressive symptoms. Patients who score 10 or greater are considered to have clinically significant depressive symptoms (positive for mild to severe depression). ${ }^{17}$ The PHQ-9 is a reliable ${ }^{17}$ and valid 17,18 scale that has been used to measure depressive symptoms in patients with HF. ${ }^{18}$ Internal consistency of the PHQ-9 in this study had a Cronbach's alpha of .83 .

Cardiac event-free survival-The outcome variable was the composite end-point of time to the first cardiac event, whether it was a cardiac-related emergency department visit, hospitalization, or death. Data on cardiac events were collected monthly by patient phone calls, hospital record review, and review of death certificates.

Variables of interest-The following sociodemographic, clinical, and psychological data were collected to completely describe the sample, compare differences in characteristics by groups, and obtain data on potential confounding variables.

Sociodemographics: Age, gender, marital status, ethnicity, and education level were collected from patient interview.

Clinical variables: New York Heart Association (NYHA) functional class was assessed by patient interview. ${ }^{19}$ Left ventricular ejection fraction (LVEF), etiology of HF, and prescribed medications were collected from the medical record and patient interview.

Psychological variable: Anxiety was assessed using the Brief Symptom Inventory (BSI) anxiety subscale. ${ }^{20}$ The anxiety subscale of the BSI consists of six items. Patients were asked to rate their level of distress on a scale from 0 (not at all) to 4 (extremely). The scores of the 6 items are summed and averaged for a total score that can range from 0 to 4 ; higher scores indicate a greater level of anxiety. The anxiety subscale of the BSI is a reliable (Cronbach's $a=.87)^{20}$ and valid scale that has been used to measure anxiety in patients with $\mathrm{HF}^{21}$ and myocardial infarction. ${ }^{20}$ For this sample, the Cronbach's a was .84 .

\section{Procedure}

This study was approved by the appropriate Institutional Review Board. All patients provided informed, signed consent. Patients' sociodemographic and clinical characteristics, anxiety, and depressive symptoms were collected by interview and medical record review at baseline. After completion of the baseline assessment, patients were given instructions on use of the MEMS bottle. Patients started to use the MEMS bottle from baseline. They returned the MEMS bottle after one or three months of continuous use of the MEMS device. Patients or family members were contacted monthly by phone to collect outcome data. 


\section{Data Management and Analysis}

All data analyses were performed using SPSS (Chicago, IL), version 17.0; a significance level of .05 was chosen to indicate statistical significance. An evidence-based cutpoint of $88 \%$ medication adherence rate by the MEMS ${ }^{11}$ was used to categorize patients into adherent and nonadherent groups. Patients were divided into presence or absence depressive symptoms groups based on the cutpoint score of 10 on the PHQ-9. We examined differences between medication adherent and nonadherent and between presence or absence depressive symptoms groups using independent t-tests and chi-square tests. Patients were further divided into 4 groups: (1) medication adherent without depressive symptoms; (2) medication adherent with depressive symptoms; (3) medication nonadherent without depressive symptoms; and (4) medication nonadherent with depressive symptoms. We used one way analysis of variance (ANOVA) with the Least Significant Difference (LSD) post hoc tests and Pearson chi-square tests to evaluate differences among the 4 groups.

The Kaplan-Meier and log-rank test was used to compare the time to cardiac event-free survival: (1) between patients in the adherent and nonadherent groups; (2) in the presence or absence of depressive symptoms; and (3) among adherent without depressive symptoms, adherent with depressive symptoms, nonadherent without depressive symptoms, and nonadherent with depressive symptoms groups. Cox proportional hazards regression modeling was used to assess the time to cardiac event-free survival between adherent and nonadherent groups, between presence or absence depressive symptoms groups, and among these four groups with and without adjusting for the following potential confounding variables: age, NYHA functional class, angiotensin converting enzyme inhibitor (ACEI) use, and anxiety (these variables were different among groups, please see Table 2). To account for differences between the two studies, we also controlled for study group (i.e., study 1 or study 2) and group assignment (i.e., usual care or intervention) in the final Cox regression models.

\section{Results}

\section{Patient Characteristics}

A total of 216 patients with HF who had complete PHQ-9 and MEMS data were included in the analysis. The mean age of patients in the sample was $60 \pm 12$ years, about one third of patients were female. The majority of the patients were Caucasian (85\%) and married (62\%). Full sample characteristics and comparison of medication adherent vs. nonadherent groups and presence or absence depressive symptoms are presented in Table 1.

\section{Medication adherence}

Of the total sample, 88 out of the 216 patients were classified as nonadherent to prescribed HF medication. As shown in the Table 1, patients in the medication nonadherent group had more depressive symptoms $(p=.018)$ and higher anxiety scores $(p=.009)$ than those in the adherent group.

\section{Depressive symptoms}

The mean of the PHQ-9 was 6.3 (standard deviation $=5.5$ ). More than one quarter $(26 \%)$ of the patients in this study had depressive symptoms. Patients with depressive symptoms were younger $(p=.003)$, had higher percentage of patients with NYHA III/IV $(p<.001)$, higher anxiety scores $(p<.001)$, and were more commonly medication nonadherent $(p=.018)$, and fewer were taking ACEI $(p=.016)$ (Table 1$)$ than patients without depressive symptoms. 


\section{Medication adherence and depressive symptoms}

When we compared the characteristics of the 4 patient groups stratified by medication adherence and depressive symptoms, there were differences in NYHA class, ACEI use, and anxiety among groups (Table 2). Patients who were medication adherent without depressive symptoms were older compared with those who were medication nonadherent with depressive symptoms $(p=.022)$. More patients who were medication nonadherent with depressive symptoms were prescribed an ACEI compared with the other groups. Regardless of medication adherence, more patients with depressive symptoms were in NYHA Class III or IV and had higher levels of anxiety than patients with no depressive symptoms.

\section{Association of medication adherence with cardiac event-free survival}

The time to the first cardiac event was significantly shorter in nonadherent patients than in adherent patients ( $p=.005$, Figure 1). In simple Cox regression modeling (Table 3 ), medication adherence predicted cardiac event-free survival (hazard ration $[H R]=2.1$ ). After controlling for study group, group assignment, age, NYHA functional class, ACEI use, and anxiety, patients who were medication nonadherent still had 1.8 times the risk of experiencing a cardiac event compared to patients who were adherent (Table 3).

\section{Association of depressive symptoms with cardiac event-free survival}

The composite endpoint of cardiac event-free survival was significantly shorter in patients with depressive symptoms than those without depressive symptoms $(p<.001$, Figure 2$)$. Depressive symptoms predicted cardiac event-free survival before and after controlling for covariates. Patients with depressive symptoms had more than twice the risk of experiencing a cardiac event compared to patients without depressive symptoms (Table 3).

\section{Combined Effect of Medication Adherence and Depressive Symptoms on Cardiac Event- free Survival}

Of the total sample, 103 (47\%) patients were classified as medication adherent without depressive symptoms, 25 (12\%) were medication adherent with depressive symptoms, 58 (27\%) were medication nonadherent without depressive symptoms, and $30(14 \%)$ were medication nonadherent with depressive symptoms. In the Kaplan-Meier and log-rank test, the composite endpoint of cardiac event-free survival was significantly worse in patients who were nonadherent with depressive symptoms than in other groups (chi-square $=29.7, p$ $<.001)$. In the Cox regression, patients who were nonadherent with depressive symptoms experienced the shortest cardiac event-free survival of all the other groups $(p<.001)$. Patients who were nonadherent with depressive symptoms had a 5 times higher risk of a cardiac event than those who were adherent without depressive symptoms $(p<.001)$. In the Kaplan-Meier and log-rank test, the composite endpoint of cardiac event-free survival was significantly worse in NYHA III/IV patients than those with NYHA I/II (chi-square $=4.238$, $p=0.04$ ). When adding study group, group assignment, age, NYHA functional class, ACEI use, and anxiety to the model, patients who were nonadherent with depressive symptoms had a 5.2 times higher risk of cardiac events than patients who were adherent without depressive symptoms (Table 4). NYHA class was no longer a significant predictor of cardiac event-free survival in the multiple Cox regression. Patients who were nonadherent without depressive symptoms or those who were adherent with depressive symptoms had a 1.2-1.3 times higher risk of a cardiac event than those who were adherent without depressive symptoms before and after controlling for covariates (Table 4). 


\section{Discussion}

This is the first study to explore the prediction of cardiac event-free survival from the combination of medication adherence and depressive symptoms in patients with HF. We found that medication adherence and depressive symptoms independently predicted cardiac event-free survival in patients with HF. Consistent with prior investigators' findings, HF patients who were adherent to prescribed medications had a lower risk of cardiac events than those who were nonadherent. ${ }^{10,11}$ Likewise, HF patients who had depressive symptoms had a higher risk of ED visits, hospitalizations, or death than those who had no depressive symptoms. 2,3

The most striking finding from this study was that medication adherence and depressive symptoms in combination predicted cardiac event-free survival in patients with HF in a synergistic fashion. In the Cox regression model, when HF patients were divided into 4 groups stratified by medication adherence and depressive symptoms, the risk of experiencing a cardiac event for patients with both risk factors (medication nonadherence and depressive symptoms) was 5 times compared to those who were medication adherent without depressive symptoms before and after adjusting for covariates; while the risk of experiencing a cardiac event for patients with only one risk factor was 1.3 times that of those with neither risk factor.

We identified three studies in which both medication adherence and depressive symptoms predicted health outcomes. ${ }^{22-24}$ Morgan and colleagues ${ }^{22,50}$ found that difficulty taking medications, an aspect of medication nonadherence, and depressive symptoms in patients with HF were independent predictors of worse health status scores on the Kansas City Cardiomyopathy Questionnaire. In a recent prospective study in 107 heart transplantation recipients, Favaro and associates ${ }^{24}$ investigated the role of major depression and medication adherence in the prediction of outcomes from cardiac transplantation and found that presence of an episode of major depression prior to cardiac transplantation indirectly predicted mortality through post-transplant malignancies, while poor medication adherence was a significant predictor of mortality. In a sample of 111 patients with HF, Moser and colleagues $^{23}$ examined whether nonadherence mediated the relationship between depression and outcomes and found that medication adherence but not dietary sodium adherence mediated the link between depression and rehospitalization/mortality. Our findings were consistent with these prior investigators' findings that both medication nonadherence and depressive symptoms predicted poor outcomes. However, the combined effect of medication adherence and depressive symptoms was not examined in these studies. Our study advances our understanding of this relationship by demonstrating that medication nonadherence and depressive symptoms in combination, predicted worse cardiac event-free survival in patients with HF before and after controlling for some important covariates. Therefore, clinicians should take both medication adherence and depressive symptoms seriously, screen/assess regularly, and should strive to provide appropriate intervention/treatment when needed.

It is well-documented that depressive symptoms predict shorter cardiac event-free survival. 2, 3, 25-29, 4, 23, 24, 25, 26 Patients with depressive symptoms were more likely to have ED visits, ${ }^{25}$ hospital readmissions, ${ }^{25,} 26,28$ and death ${ }^{26,27}$ compared with those without depressive symptoms. ${ }^{24,25}$ There are several mechanisms that could be related to the association between depressive symptoms and increased risk for ED visits, hospitalization, or death. Patients with depressive symptoms have lower heart rate variability, ${ }^{30,31}$ a higher inflammatory response, ${ }^{32}$ hyperactivity of sympathetic nervous system, greater severity of $\mathrm{HF}^{29}$ higher platelet activity, and more frequent myocardial ischemia which may explain, in part, worse outcomes ${ }^{33}$ In the current HF literature, NYHA classification has been found to be related to depressive symptoms ${ }^{34-36}$ and survival ${ }^{37,38}$ in patients with HF, and also been 
used to assess HF severity. ${ }^{39,} 40$ In our study, patients with depressive symptoms were more likely to have advanced HF as reflected by NYHA functional class compared with those without depressive symptoms. In the Kaplan-Meier and log-rank test, patients with NYHA III/IV had shorter cardiac event-free survival than those with NYHA I/II. This result is consistent with prior studies showing the impact of NYHA on rehospitalization or mortality in HF. ${ }^{37,} 38$ However, when we adjusted NYHA class in the Cox regression, NYHA class was not a predictor of cardiac event-free survival, suggesting that the observed relationship among medication adherence, depressive symptoms, and outcomes was not a result of confounding. However, we cannot rule out the possibility of unmeasured confounders (e.g., inflammatory markers) in this observational study.

In addition to these physiologic factors, medication adherence is one of the most common explanations linking depression and worse outcomes. ${ }^{3,25,27}$ Investigators have suggested that worse outcomes of patients with depressive symptoms might be associated with reduced adherence to medical therapy. ${ }^{3,25,27}$ In our study, more patients with depressive symptoms were medication nonadherent than without depressive symptoms. Our result was consistent with most prior studies that depression was associated with medication nonadherence. ${ }^{41-48}$

Many studies have focused on why depressed patients are more likely to be nonadherent than non-depressed patients. Investigators have suggested the reasons for patients with depressive symptoms are more likely to be nonadherent to their treatment regimen might due to greater feelings of hopelessness, being socially isolated, withdrawal from social network, possible reductions in the cognitive functioning that may affect memory, and no energy to carry out medical regimen. ${ }^{27,49}$ In a sample of 280 patients with HF, excessive daytime sleepiness and mild cognitive decline were 2.5 times more likely to be medication nonadherent than those without excessive daytime sleepiness or cognitive decline. ${ }^{50}$ However, we did not examine these variables in our study. Therefore, more research is needed to explore the linkages between depressive symptoms and medication adherence.

When we compared sociodemographic, clinical, and psychological variables among 4 groups (stratified by medication adherence and depressive symptoms), age, NYHA functional class, ACEI use, functional status, and anxiety were different among groups. Patients with medication nonadherent and depressive symptoms group were younger, were more likely to be NYHA III/IV class, were less likely to use ACEI, had worse functional status, and had a higher level of anxiety than those in other groups. However, when we adjusted for age, NYHA class, ACEI use, and anxiety in the Cox regression model, patients with medication nonadherence and depressive symptoms still had the highest risk of experiencing a cardiac event, suggesting that the predictive power of the combination of depressive symptoms and medication adherence for cardiac event-free survival was robust.

\section{Limitations}

Participants were recruited from one Southern tertiary hospital. Our sample included 85\% Caucasian, $65 \%$ male, and $62 \%$ married patients. Therefore, our findings warrant further study to confirm these results in a more diverse sample of patients with HF.

Second, we have small sample size within some of the 4 adherence/depression groups. Future studies of this phenomenon should include a larger number of each group, especially for medication adherent/nonadherent patients with depressive symptoms so that the complex dynamics surrounding depressive symptoms, medication adherence and outcomes can be better illuminated. Thus our findings should be considered exploratory and the need for replication emphasized. 
Third, we assessed HF severity using NYHA class instead of a more objective marker such as natriuretic peptides. However, investigators have shown plasma brain natriuretic peptide (BNP) levels were significantly increased in accordance with the NYHA class. ${ }^{51}$ And even the measure of natriuretic peptide has limitations with regard to its ability to accurately reflect severity of heart failure. For example, extreme values of plasma BNP do not correlate with the presence of $\mathrm{HF}$ or cardiomyopathy, thus limiting the usefulness of BNP as a marker of HF severity. ${ }^{52}$

Finally, medication adherence might be inflated by using the MEMS because patients knew their medication-taking behaviors were monitored (i.e., Hawthorne effect). However, the MEMS is considered a "gold standard" measure to assess medication adherence in the current adherence literature and is the closest way to get to actual behavior. ${ }^{53}$ From prior validation studies of the MEMS, serum blood levels were correlated with MEMS data indicating that patients take their medication when they open the MEMS caps and further studies have demonstrated that there was no Hawthorne effect with the use of the MEMS. ${ }^{54}$

\section{Conclusion}

The major finding of this study was that medication adherence and depressive symptoms combined to produce a synergistic effect in the prediction of cardiac event-free survival. The study highlights the importance of medication adherence and patient's depressive symptoms. Interventions to improve clinical outcomes should address both medication adherence and depressive symptoms.

\section{Acknowledgments}

This study was supported by funding from the Philips Medical-American Association of Critical Care Nurses Outcomes Grant, American Heart Association Great River Affiliate Postdoctoral Fellowship to Jia-Rong Wu, University of North Carolina at Chapel Hill Junior Faculty Development Award, University of Kentucky General Clinical Research Center (M01RR02602), grant \# R01 NR008567 from the National Institute of Nursing Research and a Center grant to the University of Kentucky, College of Nursing from NIH, NINR, 1P20NR010679. The content is solely the responsibility of the authors and does not necessarily represent the official views of the National Institute of Nursing Research or the National Institutes of Health.

\section{References}

1. Roger VL, Go AS, Lloyd-Jones DM, et al. Heart disease and stroke statistics--2011 update: A report from the american heart association. Circulation. 2011; 123:e18-e209. [PubMed: 21160056]

2. Rutledge T, Reis VA, Linke SE, et al. Depression in heart failure a meta-analytic review of prevalence, intervention effects, and associations with clinical outcomes. J. Am. CollCardiol. 2006; 48:1527-1537.

3. Faris R, Purcell H, Henein MY, et al. Clinical depression is common and significantly associated with reduced survival in patients with non-ischaemic heart failure. Eur J Heart Fail. 2002; 4:541551. [PubMed: 12167395]

4. Murray MD, Tu W, Wu J, et al. Factors associated with exacerbation of heart failure include treatment adherence and health literacy skills. Clin. Pharmacol. Ther. 2009; 85:651-658. [PubMed: 19262464]

5. Ambardekar AV, Fonarow GC, Hernandez AF, et al. Characteristics and in-hospital outcomes for nonadherent patients with heart failure: Findings from get with the guidelines-heart failure (gwtghf). Am. Heart J. 2009; 158:644-652. [PubMed: 19781426]

6. Annema C, Luttik ML, Jaarsma T. Reasons for readmission in heart failure: Perspectives of patients, caregivers, cardiologists, and heart failure nurses. Heart Lung. 2009; 38:427-434. [PubMed: 19755193] 
7. Esposito D, Bagchi AD, Verdier JM, et al. Medicaid beneficiaries with congestive heart failure: Association of medication adherence with healthcare use and costs. Am. J. Manag. Care. 2009; 15:437-445. [PubMed: 19589011]

8. Granger BB, Swedberg K, Ekman I, et al. Adherence to candesartan, placebo, outcomes in chronic heart failure in the charm programme: Double-blind, randomised, controlled clinical trial. Lancet. 2005; 366:2005-2011. [PubMed: 16338449]

9. Murray MD, Young J, Hoke S, et al. Pharmacist intervention to improve medication adherence in heart failure: A randomized trial. Ann. Intern. Med. 2007; 146:714-725. [PubMed: 17502632]

10. Wu JR, Moser DK, Chung ML, Objectively measured, but not self-reported, et al. medication adherence independently predicts event-free survival in patients with heart failure. J. Card. Fail. 2008; 14:203-210. [PubMed: 18381183]

11. Wu JR, Moser DK, De Jong MJ, et al. Defining an evidence-based cutpoint for medication adherence in heart failure. Am. Heart J. 2009; 157:285-291. [PubMed: 19185635]

12. Wu JR, Moser DK, Lennie TA, et al. Medication adherence in patients who have heart failure: A review of the literature. Nurs. Clin. North Am. 2008; 43:133-153. [PubMed: 18249229]

13. Grenard JL, Munjas BA, Adams JL, et al. Depression and medication adherence in the treatment of chronic diseases in the united states: A meta-analysis. J. Gen. Intern. Med. 2011

14. Wu JR, Corley DJ, Lennie TA, et al. Effect of a medication-taking behavior feedback theory-based intervention on outcomes in patients with heart failure. J. Card. Fail. 2012; 18:1-9. [PubMed: 22196835]

15. Wu JR, Lennie TA, De Jong MJ, et al. Medication adherence is a mediator of the relationship between ethnicity and event-free survival in patients with heart failure. J. Card. Fail. 2010; 16:142-149. [PubMed: 20142026]

16. Chung ML, Lennie TA, de Jong M, et al. Patients differ in their ability to self-monitor adherence to a low-sodium diet versus medication. J. Card. Fail. 2008; 14:114-120. [PubMed: 18325457]

17. Kroenke K, Spitzer RL. Williams JB. The phq-9: Validity of a brief depression severity measure. J. Gen. Intern. Med. 2001; 16:606-613. [PubMed: 11556941]

18. Ackermann RT, Rosenman MB, Downs SM, et al. Telephonic case-finding of major depression in a medicaid chronic disease management program for diabetes and heart failure. Gen. Hosp. Psychiatry. 2005; 27:338-343. [PubMed: 16168794]

19. Mills RM Jr. Haught WH. Evaluation of heart failure patients: Objective parameters to assess functional capacity. Clin. Cardiol. 1996; 19:455-460. [PubMed: 8790948]

20. Abu, Ruz ME.; Lennie, TA.; Riegel, B., et al. Evidence that the brief symptom inventory can be used to measure anxiety quickly and reliably in patients hospitalized for acute myocardial infarction. J. Cardiovasc. Nurs. 2010; 25:117-123. [PubMed: 20168191]

21. Khalil AA, Hall LA, Moser DK, et al. The psychometric properties of the brief symptom inventory depression and anxiety subscales in patients with heart failure and with or without renal dysfunction. Arch. Psychiatr. Nurs. 2011; 25:419-429. [PubMed: 22114796]

22. Morgan AL, Masoudi FA, Havranek EP, et al. Difficulty taking medications, depression and health status in heart failure patients. J. Card. Fail. 2006; 12:54-60. [PubMed: 16500581]

23. Moser DK, Chung ML, Riegel M, et al. Nonadherence is a mediator of the link between depressive symptoms, and rehospitalization or mortality in patients with heart failure. Circulation. 2006; 114:518.

24. Favaro A, Gerosa G, Caforio AL, et al. Posttraumatic stress disorder and depression in heart transplantation recipients: The relationship with outcome and adherence to medical treatment. Gen. Hosp. Psychiatry. 2011; 33:1-7. [PubMed: 21353121]

25. Himelhoch S, Weller WE, Wu AW, et al. Chronic medical illness, depression and use of acute medical services among medicare beneficiaries. Med. Care. 2004; 42:512-521. [PubMed: 15167319]

26. Jiang W, Alexander J, Christopher E, et al. Relationship of depression to increased risk of mortality and rehospitalization in patients with congestive heart failure. Arch. Intern. Med. 2001; 161:1849-1856. [PubMed: 11493126]

27. Junger J, Schellberg D, Muller-Tasch T, et al. Depression increasingly predicts mortality in the course of congestive heart failure. Eur J Heart Fail. 2005; 7:261-267. [PubMed: 15701476] 
28. Sullivan MD, Levy WC, Crane BA, et al. Usefulness of depression to predict time to combined end point of transplant or death for outpatients with advanced heart failure. Am. J. Cardiol. 2004; 94:1577-1580. [PubMed: 15589024]

29. Vaccarino V, Kasl SV, Abramson J, et al. Depressive symptoms and risk of functional decline and death in patients with heart failure. J. Am. Coll. Cardiol. 2001; 38:199-205. [PubMed: 11451275]

30. Francis JL, Weinstein AA, Krantz DS, et al. Association between symptoms of depression and anxiety with heart rate variability in patients with implantable cardioverter defibrillators. Psychosom. Med. 2009; 71:821-827. [PubMed: 19661191]

31. Kemp AH, Quintana DS, Gray MA, et al. Impact of depression and antidepressant treatment on heart rate variability: A review and meta-analysis. Biol. Psychiatry. 2010; 67:1067-1074. [PubMed: 20138254]

32. Dentino AN, Pieper CF, Rao MK, et al. Association of interleukin- 6 and other biologic variables with depression in older people living in the community. J. Am. Geriatr. Soc. 1999; 47:6-11. [PubMed: 9920223]

33. Musselman DL, Evans DL, Nemeroff CB. The relationship of depression to cardiovascular disease: Epidemiology, biology, and treatment. Arch. Gen. Psychiatry. 1998; 55:580-592. [PubMed: 9672048]

34. Altay H, Zorlu A, Kocum HT, et al. Relationship between parathyroid hormone and depression in heart failure. Arq. Bras. Cardiol. 2012; 99:915-923. [PubMed: 22983154]

35. Rollman BL, Herbeck Belnap B, Mazumdar S, et al. A positive 2-item patient health questionnaire depression screen among hospitalized heart failure patients is associated with elevated 12-month mortality. J. Card. Fail. 2012; 18:238-245. [PubMed: 22385945]

36. Suzuki T, Shiga T, Kuwahara K, et al. Prevalence and persistence of depression in patients with implantable cardioverter defibrillator: A 2-year longitudinal study. Pacing Clin. Electrophysiol. 2010; 33:1455-1461. [PubMed: 20946285]

37. Pocock SJ, Ariti CA, McMurray JJ, et al. Predicting survival in heart failure: A risk score based on 39372 patients from 30 studies. Eur. Heart J. 2012

38. Son YJ, Lee Y, song EK. Adherence to a sodium-restricted diet is associated with lower symptom burden and longer cardiac event-free survival in patients with heart failure. J. Clin. Nurs. 2011; 20:3029-3038. [PubMed: 21707808]

39. Gottlieb SS, Kop WJ, Ellis SJ, et al. Relation of depression to severity of illness in heart failure (from heart failure and a controlled trial investigating outcomes of exercise training [hf-action]). Am. J. Cardiol. 2009; 103:1285-1289. [PubMed: 19406273]

40. Faller H, Stork S, Schuler M, et al. Depression and disease severity as predictors of health-related quality of life in patients with chronic heart failure--a structural equation modeling approach. J. Card. Fail. 2009; 15:286-292. e282. [PubMed: 19398075]

41. Krousel-Wood M, Islam T, Muntner P, et al. Association of depression with antihypertensive medication adherence in older adults: Cross-sectional and longitudinal findings from cosmo. Ann. Behav. Med. 2010; 40:248-257. [PubMed: 20703839]

42. Gehi A, Haas D, Pipkin S, et al. Depression and medication adherence in outpatients with coronary heart disease: Findings from the heart and soul study. Arch. Intern. Med. 2005; 165:2508-2513. [PubMed: 16314548]

43. Farrell K, Shen BJ, Mallon S, et al. Utility of the millon behavioral medicine diagnostic to predict medication adherence in patients diagnosed with heart failure. J Clin Psychol Med Settings. 2011; 18:1-12. [PubMed: 21222020]

44. Hansen RA, Dusetzina SB, Song L, et al. Depression affects adherence measurement but not the effectiveness of an adherence intervention in heart failure patients. J Am Pharm Assoc (2003). 2009; 49:760-768. [PubMed: 19926556]

45. Cukor D, Rosenthal DS, Jindal RM, et al. Depression is an important contributor to low medication adherence in hemodialyzed patients and transplant recipients. Kidney Int. 2009; 75:1223-1229. [PubMed: 19242502]

46. Julian LJ, Yelin E, Yazdany J, et al. Depression, medication adherence and service utilization in systemic lupus erythematosus. Arthritis Rheum. 2009; 61:240-246. [PubMed: 19177526] 
47. DiMatteo MR, Lepper HS. Depression is a risk factor for noncompliance with medical treatment: Meta-analysis of the effects of anxiety and depression on patient adherence. Arch. Intern. Med. 2000; 160:2101-2107. [PubMed: 10904452]

48. Glassman AH, Bigger JT Jr. Gaffney M. Psychiatric characteristics associated with long-term mortality among 361 patients having an acute coronary syndrome and major depression: Sevenyear follow-up of sadhart participants. Arch. Gen. Psychiatry. 2009; 66:1022-1029. [PubMed: 19736359]

49. Wing RR, Phelan S, Tate D. The role of adherence in mediating the relationship between depression and health outcomes. J. Psychosom. Res. 2002; 53:877-881. [PubMed: 12377297]

50. Riegel B, Moelter ST, Ratcliffe SJ, et al. Excessive daytime sleepiness is associated with poor medication adherence in adults with heart failure. J. Card. Fail. 2011; 17:340-348. [PubMed: 21440873]

51. Nakamura T, Funayama H, Kubo N, et al. Association of hyperadiponectinemia with severity of ventricular dysfunction in congestive heart failure. Circ J. 2006; 70:1557-1562. [PubMed: 17127799]

52. Law C, Glover C, Benson K, et al. Extremely high brain natriuretic peptide does not reflect the severity of heart failure. Congest Heart Fail. 2010; 16:221-225. [PubMed: 20887619]

53. Cramer JA. Microelectronic systems for monitoring and enhancing patient compliance with medication regimens. Drugs. 1995; 49:321-327. [PubMed: 7774509]

54. Kimmel SE, Chen Z, Price M, et al. The influence of patient adherence on anticoagulation control with warfarin: Results from the international normalized ratio adherence and genetics (in-range) study. Arch. Intern. Med. 2007; 167:229-235. [PubMed: 17296877] 


\section{Medication Adherence on Cardiac Event-free Survival}

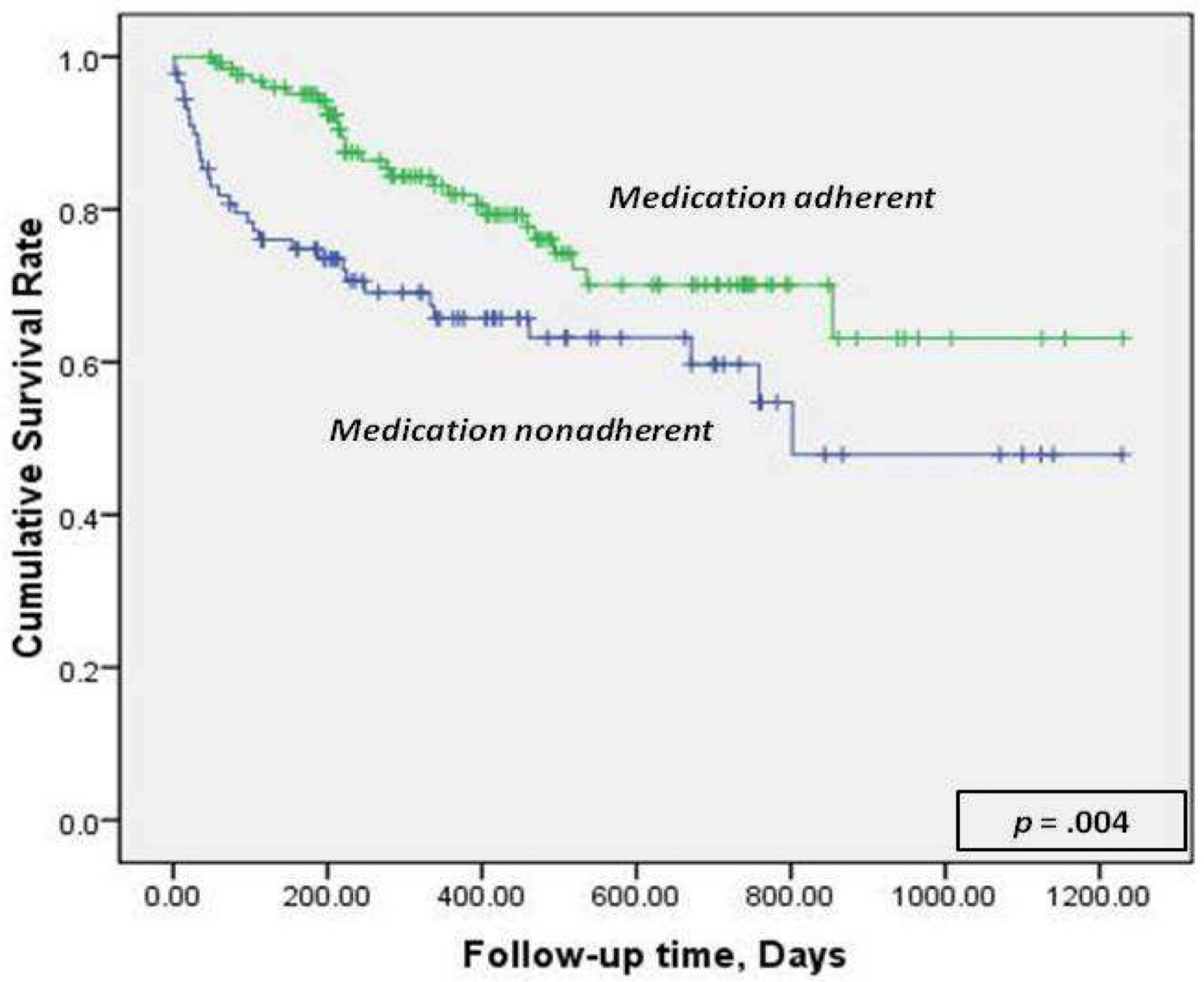

Figure 1.

Medication adherence and cardiac event-free survival 


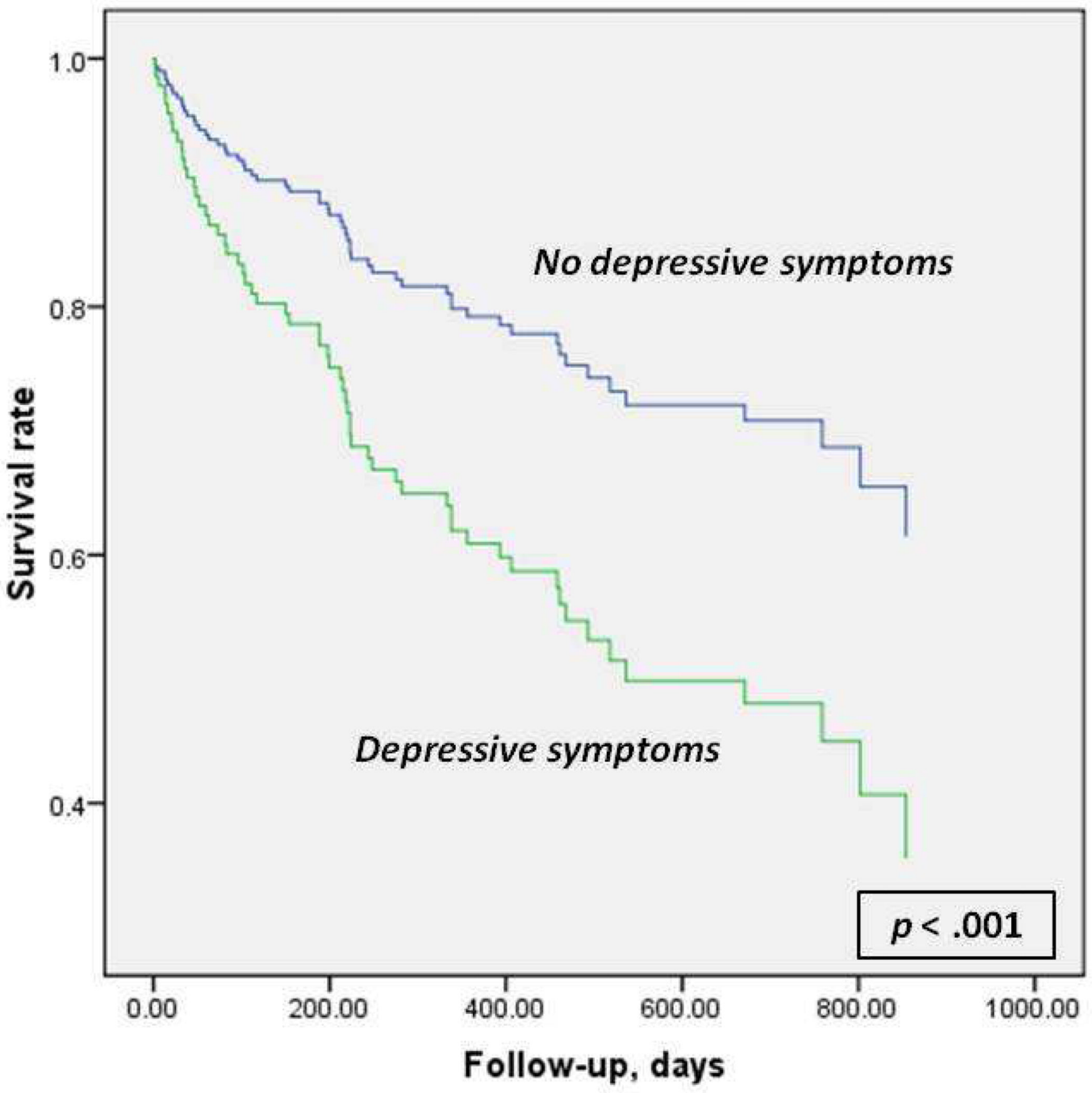

Figure 2.

Depressive symptoms and cardiac event-free survival 


\section{Cardiac Event-free Survival for 4 Groups}

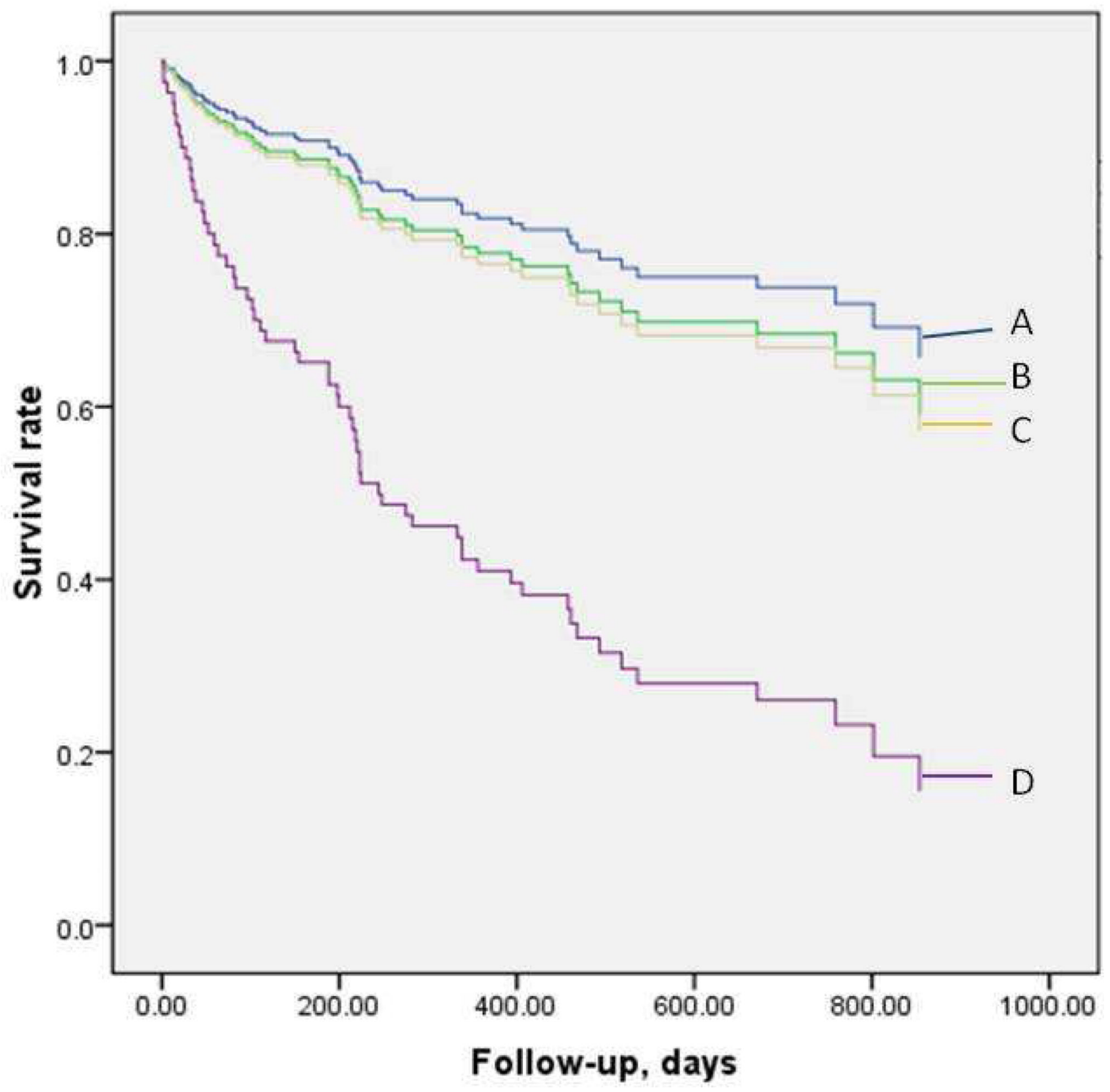

Figure 3.

Cardiac event-free survival for patients stratified by medication adherence and depressive symptoms.

A: Medication adherent without depressive symptoms

B: Medication adherent with depressive symptoms

$\mathrm{C}$ : Medication nonadherent without depressive symptoms

D: Medication nonadherent with depressive symptoms 


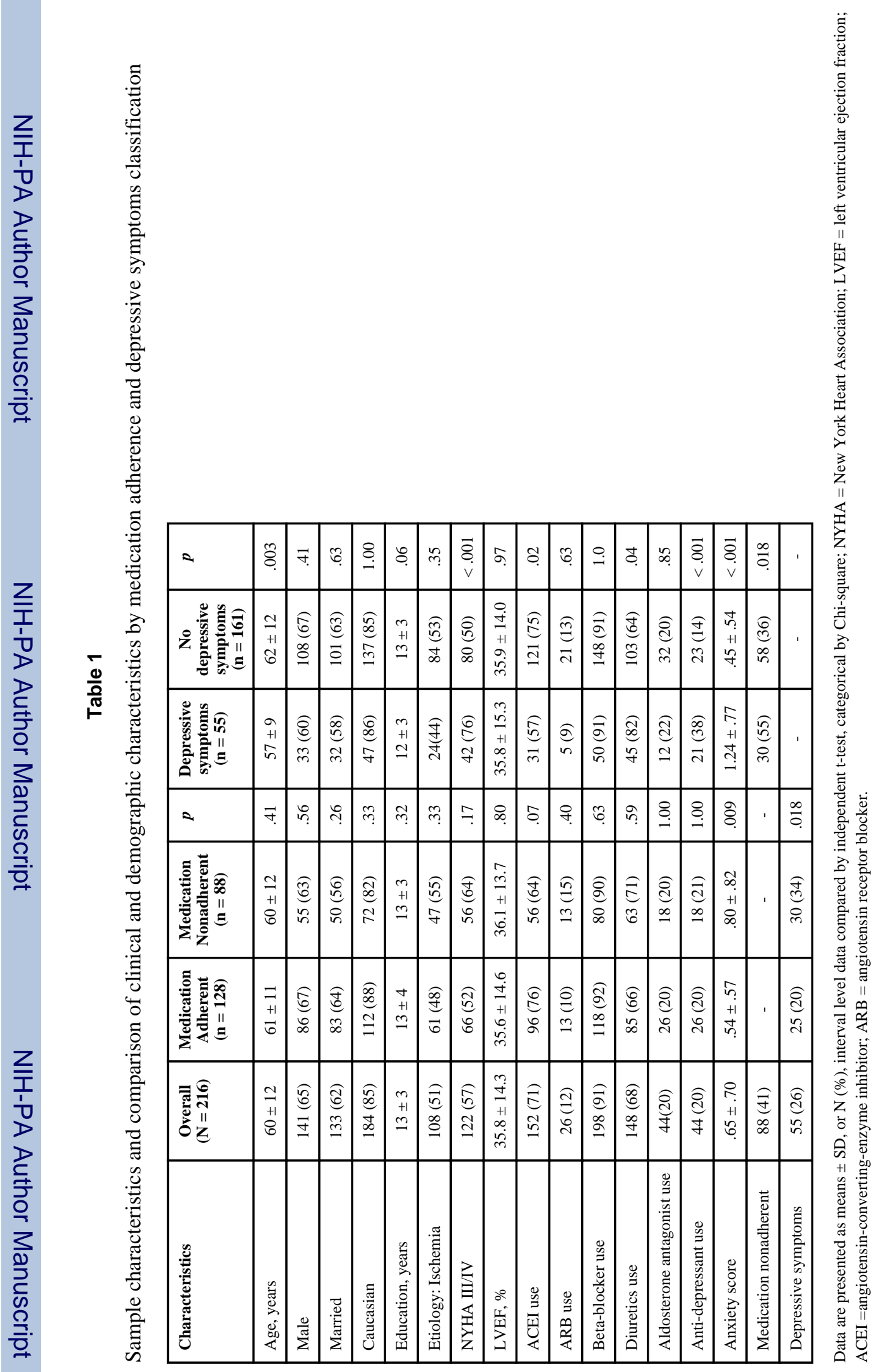




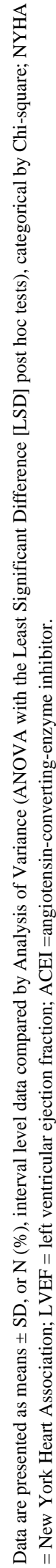

J Card Fail. Author manuscript; available in PMC 2014 May 01. 
Table 3

Cox regression modeling: medication adherence alone and depressive symptoms alone on Cardiac Event-free Survival $(\mathrm{N}=216)$

\begin{tabular}{|l|c|c|c|}
\hline Variables & Hazard Ratio & 95\% CI & $p$ \\
\hline Simple Cox Regression: without adjusting & & & \\
\hline Medication adherence $^{*}$ & & & \\
\hline Medication adherent & 1.0 & - & - \\
\hline Medication nonadherent & 2.07 & $1.242-3.462$ & .005 \\
\hline Depressive symptoms $^{* *}$ & & & \\
\hline No depressive symptoms $^{*}$ & 1.0 & - & - \\
\hline Depressive symptoms & 2.65 & $1.568-4.489$ & $<.001$ \\
\hline Cox Regression: adjusting for covariates $^{\dagger}$ & & & \\
\hline Medication adherence $^{*}$ & & & \\
\hline Medication adherent & 1.0 & - & - \\
\hline Medication nonadherent & 1.80 & $1.065-3.042$ & .028 \\
\hline Depressive symptoms $^{\xi}$ & & & \\
\hline No depressive symptoms & 1.0 & - & - \\
\hline Depressive symptoms & 2.404 & $1.255-4.606$ & .008 \\
\hline
\end{tabular}

CI: confidence interval

chi-square $=8.117 ; p=.004$;

**

chi-square $=14.278 ; p<.001$

${ }^{\dagger}$ Controlling for study group, group assignment, age, New York Heart Association functional class, and angiotensin-converting-enzyme inhibitor use.

${ }_{\text {chi-square }}=19.187 ; p=.008$

$\xi_{\text {chi-square }}=22.02 ; p=.003$ 


\section{Table 4}

Cox regression modeling: combined effects of medication adherence and depressive symptoms on Cardiac Event-free Survival $(\mathrm{N}=216)$

\begin{tabular}{|l|c|c|c|}
\hline Variables & Hazard Ratio & $\mathbf{9 5 \%}$ CI & $p$ \\
\hline Simple Cox Regression: without adjusting ${ }^{*}$ & & & \\
\hline Medication adherent without depressive symptoms & 1.0 & - & - \\
\hline Medication adherent with depressive symptoms & 1.41 & $.568-3.5$ & .46 \\
\hline Medication nonadherent without depressive symptoms & 1.366 & $.704-2.652$ & .14 \\
\hline Medication nonadherent with depressive symptoms & 4.949 & $2.6-9.423$ & $<.001$ \\
\hline Cox Regression: adjusting for covariates ${ }^{* *}$ & & & \\
\hline Step 1 & & & \\
\hline Study group & 1.447 & $.752-2.786$ & .27 \\
\hline Group assignment & 2.577 & $1.085-6.119$ & .03 \\
\hline Step 2 & 1.003 & $.980-1.026$ & .82 \\
\hline Age & 1.291 & $.863-1.932$ & .21 \\
\hline NYHA & .757 & $.429-1.334$ & .34 \\
\hline ACEI use & 1.412 & $1.003-1.988$ & .048 \\
\hline Anxiety & 1.302 & $.492-3.445$ & .60 \\
\hline Step 3 & 1.227 & $.625-2.407$ & .55 \\
\hline Medication adherent and no depressive symptoms & 1.0 & $2.318-11.551$ & $<.001$ \\
\hline Medication adherent and depressive symptoms & \multicolumn{1}{|l}{} \\
\hline Medication nonadherent and no depressive symptoms & & - & - \\
\hline Medication nonadherent and depressive symptoms & 5.175 & & \\
\hline
\end{tabular}

CI: confidence interval

chi-square $=30.537, p<.001$

**

chi-square $=37.818, p<.001$

NYHA=New York Heart Association functional class, ACEI=angiotensin-converting-enzyme inhibitor. 